\title{
Broadband and high resolution measurements of cavity loss and dispersion
}

\author{
Dominik Charczun $^{1 *}$, Grzegorz Kowzan ${ }^{1}$, Agata Cygan ${ }^{1}$, Ryszard S. Trawiński ${ }^{1}$, Daniel Lisak ${ }^{1}$, Piotr Masłowski ${ }^{1}$ \\ ${ }^{1}$ Institute of Physics, Faculty of Physics, Astronomy and Informatics, Nicolaus Copernicus University in Toruń, \\ Grudziądzka 5, 87-100 Toruń
}

Received June 03, 2018; accepted June 29, 2018; published June 30, 2018

\begin{abstract}
We present a method for broadband measurements of dispersion and loss of an optical cavity. We employ an optical frequency comb directly coupled into a cavity to scan cavity modes and retrieve their shapes and positions. Measurement data are acquired using instrumental-line-shape-free Fourier transform spectrometry. This method can be developed into a powerful tool for optical loss and dispersion measurements concerning broadband characterization of optical elements as well as absorption and dispersion spectroscopy.
\end{abstract}

The invention of an optical frequency comb (OFC) revolutionized many fields of science through its numerous applications, including frequency metrology [1], astronomy [2] and breath analysis [3]. Exceptional frequency precision and accuracy provided by the OFC are its outstanding features exploited for spectroscopic measurements, both as reference for other methods [4] as well as direct use for measurements [5].

The OFC consists of equidistant narrow laser lines separated by $f_{\text {rep }}$ frequency and offset from zero frequency by $f_{0}$. Optical cavities share a similar mode structure of almost equidistant cavity modes. This creates a possibility to match the comb teeth to cavity modes and achieve broadband simultaneous transmission in a mirror dispersion-limited range, taking advantage of an enhanced optical path through the sample [5].

In this work our goal was to prove that an optical frequency comb combined with a Fourier transform spectrometer can be used to quickly assess loss and dispersion in a high-finesse optical cavity in a wide spectral range by measuring widths and positions of cavity modes. The obtained precision of cavity mode-width measurements is an order of magnitude better than earlier comb-based results [6].

The experimental setup is shown in Fig. 1. A continuous wave (CW) laser is locked to the cavity using the well-known Pound-Drever-Hall method [7]. A fraction of the $\mathrm{CW}$ light is used to create a heterodyne signal with one of the comb teeth, which is mixed with a reference frequency to create an error signal. The signal acts on the comb repetition rate in such a way that it phase-locks the comb tooth to the laser. This way an indirect lock to the cavity is achieved as the $\mathrm{CW}$ laser

*E-mail: charczun@ fizyka.umk.pl works as an intermediary between the comb and the cavity. The other degree of freedom of the OFC, which is the carrier-envelope offset frequency $\left(f_{0}\right)$, is locked to an external frequency reference. This locking scheme makes it possible to tune the comb modes and scan the shapes of the cavity modes overcoming the limitation of transmitted bandwidth induced by cavity dispersion. The measurement procedure requires obtaining multiple interferograms, each for a different $\mathrm{CW}-\mathrm{OFC}$ heterodyne beatnote frequency, corresponding to different relative detuning of the OFC and cavity modes. Moreover, having acquired the cavity mode shapes, one can obtain information about molecular absorption and dispersion from their widths and positions. This approach, which has been previously shown both with a CW laser [8] and with the OFC [6], which offers parallel acquisition of thousands of cavity modes simultaneously.

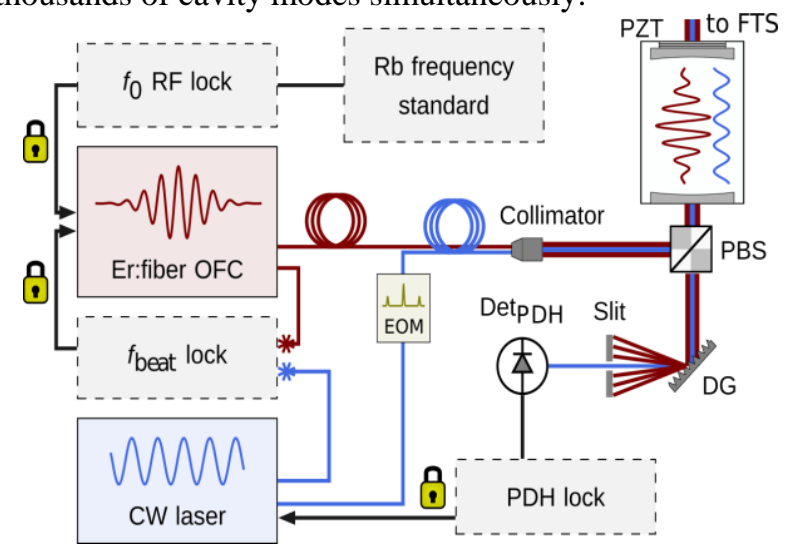

Fig. 1. Experimental setup. DG - diffraction grating, EOM - electrooptical modulator, $f_{0}$ - offset frequency of the OFC, $f_{\text {beat }}$ - frequency of the beat note between one of the comb teeth and the CW laser, FTS Fourier transform spectrometer, OFC - optical frequency comb, PBS polarizing beam splitter, DetPDH - a photodiode detector for the Pound-

Drever-Hall method, PZT - piezoelectric transducer, $\mathrm{RF}$ - radio frequency.

The obvious requirement for measurement of cavity mode shapes is to resolve the broadband comb spectrum. In this work we employ a Fourier transform spectrometer (FTS) based on a Michelson interferometer. Those devices, however, have an inherent resolution limitation caused by the instrumental line-shape (ILS). The ILS 
distorts the measured spectral features if their width is of the order or below the nominal resolution of the spectrometer, given by the inverse of an attained optical path difference. This limitation is overcome by fulfilling the condition:

$$
O P D=c / f_{\text {rep }}
$$

where OPD is the optical path difference between the interferometer arms, $c$ is the speed of light and $f_{\text {rep }}$ is the repetition rate of the OFC. This causes the zero crossings of the ILS to occur with a period of $f_{\text {rep }}$ [9]. This way the retrieved comb teeth intensities are not affected by the ILS of adjacent teeth and samples of the discrete Fourier transform of the digitized interferogram occur exactly at the comb teeth frequencies. The deleterious effect of the ILS is nullified, allowing higher spectral resolution (so called sub-nominal resolution) than in standard FTS [9, $10]$.

To ensure fast measurement time we decided to employ the Vernier scheme of cavity-mode filtering [11]. In our case, the repetition rate of the comb was $250 \mathrm{MHz}$. The free spectral range of the cavity was set to $266.7 \mathrm{MHz}$, or $16 / 15$ times $f_{\text {rep. }}$. This way only every sixteenth comb tooth is superimposed with a cavity mode and transmitted, yielding a new repetition rate of $4 \mathrm{GHz}$ behind the cavity. This spacing is sufficient for broadband mirror dispersion assessment or measurement of spectra of large molecules, while significantly decreasing the required OPD. In this case the required OPD to ensure correct sampling was equal to $c / 16 f_{\text {rep }}$, or $\sim 7.48 \mathrm{~cm}$. The actual OPD we used in this measurement was equal to $22.445386(32) \mathrm{cm}$, giving us the sample spacing of $\sim 1.33 \mathrm{GHz}$, or two points between two consecutive comb modes, which allowed clear differentiation of the comb teeth. In the Figures, every third point was plotted. The OPD was measured with a HeNe laser in the setup. The total time of data acquisition in this measurement was 83 seconds, however some additional time is needed for tuning, data operations and additional cart travel distance due to finite acceleration.

The acquired interferograms are Fourier-transformed (examples shown in Fig. 2a) and interleaved to obtain the shapes of cavity modes in a wide spectral range. Then each cavity mode is located and a Lorentzian fit is performed (Fig. 2b), from which positions, widths and amplitudes of cavity modes are obtained. In this measurement the cavity was filled with 122 Torr of a mixture of $\mathrm{N}_{2}$ and $1000 \mathrm{ppm} \mathrm{CO}$.

Fitted cavity mode widths can be used to obtain the values of the light loss in the cavity and its finesse, similarly to the decay constant measured in a cavity ringdown (CRD) method [12]. The value of finesse for the frequency of cavity mode in Fig. $2 b$ was $19290 \pm 156$ while the finesse obtained from the mean width of modes in the range $6350-6375 \mathrm{~cm}^{-1}$ was $19080 \pm 370$. For comparison, we performed a CRD measurement with the same CW laser used for the beatnote with the OFC. The measured value of finesse obtained in this way was $19020 \pm 312$. All obtained values are in agreement with each other within the stated uncertainties equal to 1 standard deviation values.
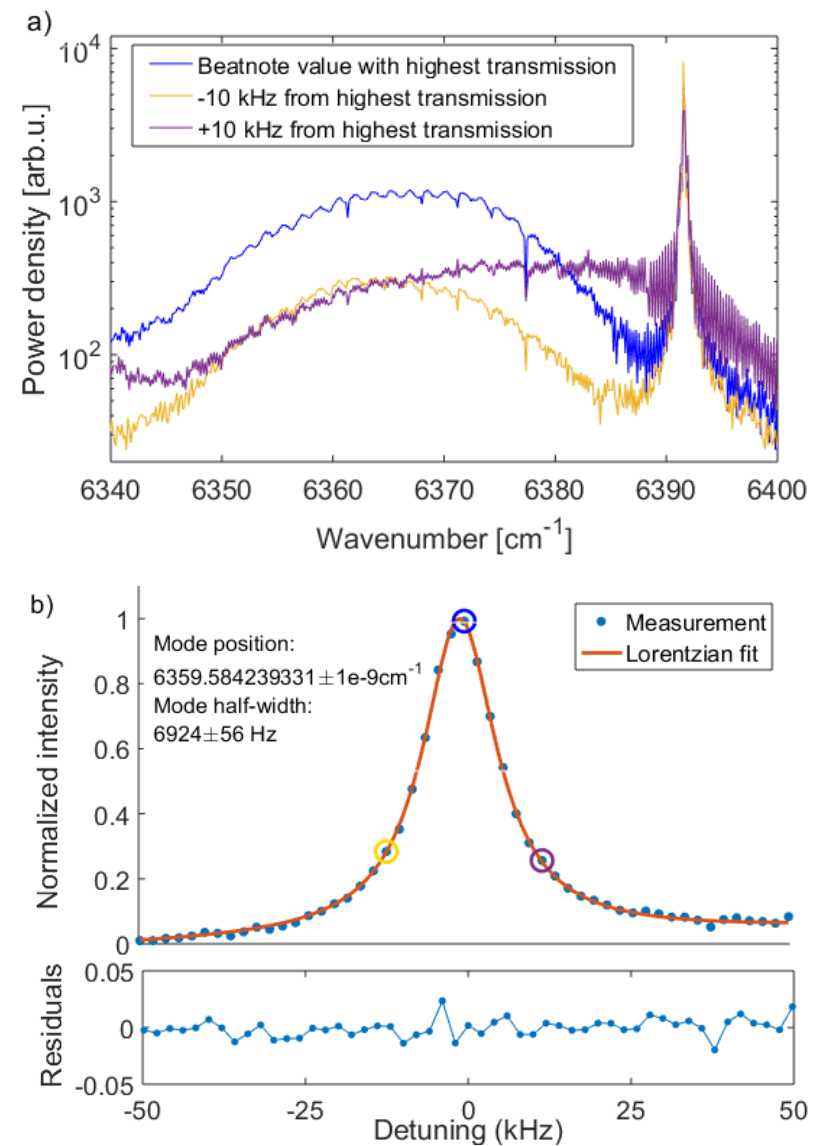

Fig. 2. a) Three interleaved OFC transmission spectra taken with different beatnote values. The blue plot is the highest transmission spectrum, yellow and purple spectra are taken with the CW-OFC beatnote tuned by $-10 \mathrm{kHz}$ and $+10 \mathrm{kHz}$ respectively. The sharp peak on the right is the CW laser distorted by the residual ILS. b) Close-up of a single measured cavity mode with superimposed Lorentzian curve fit. Error values are equal to 1 standard deviation. Single points from the spectra as shown in panel a) were used to reconstruct the shape of the cavity mode and are distinguished by colored circles.

Figure 3 contains the spectrum of cavity mode shifts relative to the position of the nearest comb tooth. The OFC in this case serves as a ruler of equidistant points creating a reference frequency axis, as the positions of the comb modes are not dependent on dispersion. This approach is equivalent to choosing one of the cavity modes as a reference point and applying the Vernieradjusted $f_{\text {rep }}$ as a shift to create the reference frequency axis. Since the value of GDD is based on a second derivative of the shift [6], this choice does not influence 
its determination. The outlying points probe molecular transitions. The orange curve is a sum of a fitted $3^{\text {rd }}$ degree polynomial and calculated molecular dispersion spectrum.

It is apparent that the calculated positions of the lines, based on the HITRAN database [13] agree with the positions of spectral features visible in the measured spectrum, but much denser sampling would be required to retrieve their line shapes. Nevertheless, even the presence of an absorber such as $\mathrm{CO}$ does not prevent the retrieval of a mirror cavity dispersion spectrum. In Fig. 4 we present the group delay dispersion (GDD) calculated from the polynomial fitted to mode positions as described in [8]. The error is calculated from polynomial parameter uncertainties and scales from $0.08 \mathrm{fs}^{2}$ in the center to 0.14 $\mathrm{fs}^{2}$ on the edges of the measurement range. The error is increased by the presence of molecular dispersion, even though the most shifted cavity modes were excluded from the fit.

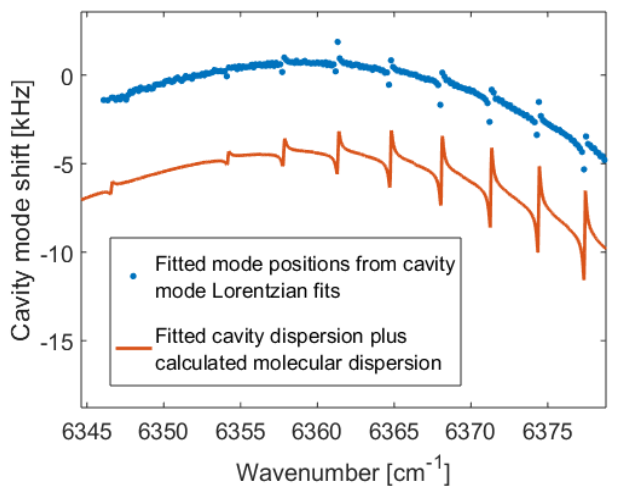

Fig. 3. Blue: spectrum of fitted relative cavity mode shifts from the position of a chosen reference mode. Orange: a sum of fitted cavity dispersion and calculated molecular dispersion of $\mathrm{CO}$, shifted by $-5 \mathrm{kHz}$ for clarity.

In conclusion, we performed a fast and precise measurement of cavity losses and dispersion in the range of around $40 \mathrm{~cm}^{-1}$. Our results confirm the claims presented in [9] and [10], that the resolution of a Fourier transform spectrometer, when an OFC light is used, is not limited by the OPD, but by the comb line width provided that the described method is utilized.

The reported results open the possibility of performing more advanced, broadband measurements of absorption and dispersion spectra to be applied in precise characterization of optical elements, accurate measurements of the refractive index of gases and potentially molecular spectroscopy.

The research was supported by the National Science Center, Poland, project No. 2015/18/E/ST2/00585,
2016/21/N/ST2/00334, 2016/23/B/ST2/00730. G.K. was supported by National Science Center, Poland scholarship 2017/24/T/ST2/00242. The research is part of the program of the National Laboratory FAMO in Torun, Poland.

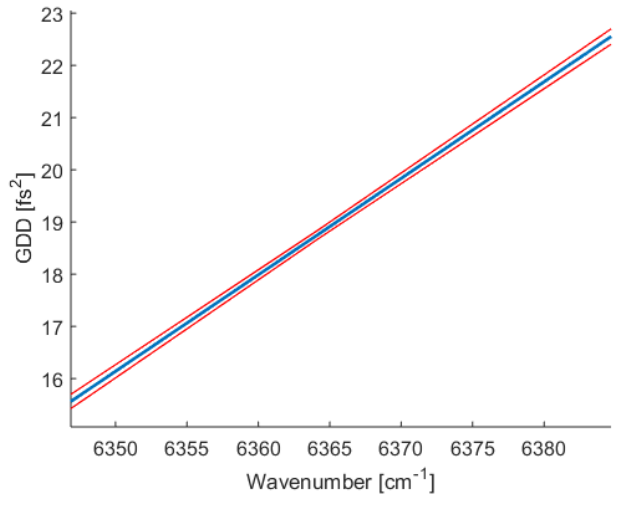

Fig. 4. Blue: group delay dispersion spectrum calculated from the polynomial fitted to relative cavity mode positions. Red: calculated error margins.

\section{References}

[1] Th. Udem, R. Holzwarth, T.W. Hänsch, Nature 416, 233 (2002).

[2] R.A. McCracken, J.M. Charsley, D.T. Reid, Opt. Expr. 25(13), 15058 (2017)

[3] M.J. Thorpe, D. Balslev-Clausen, M.S. Kirchner, J. Ye, Opt. Expr. 16(4), 2387 (2008)

[4] J. Domysławska et al., J. Chem. Phys. 136, 024201 (2012).

[5] P. Masłowski K.C. Cossel, A. Foltynowicz, J. Ye. in G. Gagliardi and H.-P. Loock, Cavity-Enhanced Spectroscopy and Sensing (Springer 2014).

[6] L. Rutkowski et al., Opt. Expr. 25(18), 21711 (2017).

[7] E.D. Black, Amer. J. Phys. 69, 79 (2001).

[8] A. Cygan et al., Opt. Expr. 23, 14472 ( 2015).

[9] P. Masłowski et al., Phys. Rev. A 93, 021802(R) (2016).

[10] L. Rutkowski, P. Masłowski, A.C. Johansson, A. Khodabakhsh, A. Foltynowicz, J. Quant. Spect. Rad. Trans. 204, 63 (2018).

[11] S. Schiller, Opt. Lett. 27, 766 (2002).

[12] A. Cygan et al., J. Chem. Phys. 144, 214202-1 (2016).

[13] I.E. Gordon et al., J. Quant. Spect. Radiat. Trans. 111, 1317 (2017). 